\title{
GSH depletion and consequent AKT inhibition contribute to the Nrf2 knockdown-induced decrease in proliferation in glioblastoma $\mathrm{U} 251$ cells
}

\author{
YUE JIA $^{1}$, HAN-DONG WANG ${ }^{1}$, QIANG WANG ${ }^{1}$, HUI DING $^{2}$, HE-MING WU ${ }^{3}$ and HAO PAN ${ }^{1}$ \\ ${ }^{1}$ Department of Neurosurgery, Jinling Hospital, School of Medicine, Nanjing University, Nanjing, Jiangsu 210002; \\ ${ }^{2}$ Department of Neurosurgery, Jinling Hospital, School of Medicine, Southern Medical University (Guangzhou), Nanjing, \\ Jiangsu 210002; ${ }^{3}$ Department of Neurosurgery, Nanjing Jingdu Hospital, Nanjing, Jiangsu 210002, P.R. China
}

Received July 18, 2016; Accepted October 12, 2016

DOI: $10.3892 /$ or.2017.5467

\begin{abstract}
Nuclear factor erythroid 2-related factor 2 (Nrf2), a pivotal transcription regulator that controls the expression of numerous antioxidant and cytoprotective genes, was recently defined as a proto-oncogene. However, the role and mechanism of Nrf2 in glioma pathoetiology remain unclear. In the present study, we first evaluated the expression patterns of Nrf2 in normal human astrocytes and 3 glioblastoma (GBM) cell lines (U251, U87 and A172) and found that all 3 GBM cell lines overexpressed Nrf2, with the highest level observed in the U251 cells. We further assessed the biological effects of Nrf2 in U251 cells by specific knockdown of Nrf2 using lentivirus-mediated RNA interference. We discovered that Nrf2 deficiency led to a decrease in U251 cell proliferation and caused intracellular redox imbalance [diminished glutathione (GSH) levels and increased reactive oxygen species (ROS) levels]. Both $N$-acetylcysteine and glutathione monoethyl ester (GMEE) supplementation completely eliminated the increased levels of ROS that were present in the Nrf2-deficient U251 cells. However, only GMEE supplementation both reversed Nrf2 deficiency-induced cell growth arrest and restored intracellular GSH levels. Moreover, AKT and ERK1/2
\end{abstract}

Correspondence to: Professor Han-Dong Wang, Department of Neurosurgery, Jinling Hospital, School of Medicine, Nanjing University, 305 East Zhongshan Road, Nanjing, Jiangsu 210002, P.R. China

E-mail: njhdwang@hotmail.com

Abbreviations: AKT, protein kinase B; DCF, dichlorofluorescein; ERK1/2, extracellular signal-regulated kinase 1/2; GBM, glioblastoma; GCLC, catalytic subunit of $\gamma$-glutamylcysteinyl ligase; GMEE, glutathione monoethyl ester; GR, glutathione reductase; GSH, glutathione; HO-1, heme oxygenase-1; NAC, $\mathrm{N}$-acetylcysteine; NHA, normal human astrocytes; NQO1, NAD(P)H:quinone oxidoreductase 1; Nrf2, nuclear factor erythroid 2-related factor 2; ROS, reactive oxygen species; shRNA, short hairpin RNA; STAT3, signal transducers and activation of transcription 3

Key words: Nrf2, glioblastoma, glutathione, AKT, cell proliferation signaling were both impaired in the Nrf2-knockdown U251 cells, but GMEE supplementation restored AKT signaling but not ERK1/2 signaling, and blocking AKT signaling with an AKT-specific inhibitor greatly diminished the GMEEinduced Nrf2-deficient cell proliferation. In conclusion, our findings revealed novel functions for Nrf2 in the regulation of redox status and cell proliferation, and that intracellular GSH levels and AKT signaling are required for this process, a new viewpoint by which to comprehend the role and underlying mechanism of Nrf2 in tumorigenesis.

\section{Introduction}

Oxidative stress in cancer is one of the most important metabolic stresses caused by both extrinsic (reperfusion of a hypoxic microenvironment) and intrinsic (uncoupled mitochondrial activity) factors $(1,2)$. From the viewpoint of carcinogenesis, oxidative stress is a double-edged sword, since it promotes genetic alterations through DNA damage to drive malignant progression while also causing cell damage and inducing apoptosis $(3,4)$. Under physiological conditions, cells possess molecular mechanisms that enable them to adapt to these stresses. In multicellular organisms, adaptive responses to oxidative stresses are regulated by nuclear factor erythroid 2-related factor 2 (Nrf2), a master transcription factor of many antioxidant genes and phase II detoxifying enzymes (5). Moreover, recent studies have demonstrated that cancer cells can also use Nrf2 and its target genes to adapt to multiple stresses, which is beneficial for cancer cell survival, leading to malignant progression and lethal characteristics such as invasion, metastasis and chemotherapy resistance (6). Therefore, some researchers have pointed out that under these conditions, Nrf2 can be defined as a proto-oncogene.

Transcription factor Nrf2, a critical element in the survival of healthy cells in response to oxidative stress, belongs to the Keap1-Nrf2-antioxidant response element (ARE) signaling pathway and upregulates many ARE-containing genes such as NAD(P)H:quinone oxidoreductase 1 (NQO1), heme oxygenase-1 (HO-1), aldo-keto reductase 1C1 (AKR1C1) and thioredoxin ( $\operatorname{Tr} x)(7)$. In addition to these enzymes, these proteins also include, for example, those involved in the 
biosynthesis and regeneration of glutathione (GSH), a very effective scavenger of reactive oxygen species (ROS) and electrophiles, such as heavy and light chains of $\gamma$-glutamyl cysteinyl ligase (8), the $\mathrm{x}-\mathrm{CT}$ (subunit of the cystine/glutamate transporter) component of the cystine/glutamate exchange transport system (9), the glutathione reductase (GR) and the GSH synthetase $(10,11)$.

In the present study, we first assessed the expression patterns of Nrf2 in normal human astrocytes and 3 GBM cell lines, and subsequently silenced Nrf2 expression in the GBM cell line U251 using RNA interference technology. Functional analysis demonstrated that Nrf2 knockdown led to a decrease in U251 cell proliferation, as well as resulted in intracellular redox imbalance (decreased levels of GSH and increased levels of ROS). In addition, suppression of Nrf2 also resulted in the impairment of the AKT and ERK1/2 signaling pathways. Finally, we ascertained that exogenous supplementation of Nrf2-knockdown cells with glutathione monoethyl ester (GMEE; a membrane-permeable derivative of GSH that can supplement cellular GSH levels) restored their intracellular GSH levels, cell proliferation defects and AKT inhibition, confirming that GSH depletion and AKT pathway inhibition are critical for GBM cell proliferation. Our findings may shed light on the role and underlying mechanism of Nrf2 in the regulation of glioma proliferation via GSH depletion and consequent AKT inhibition, a new viewpoint by which to comprehend the role and mechanism of $\mathrm{Nrf} 2$ in glioma pathoetiology.

\section{Materials and methods}

Reagents and antibodies. $N$-acetylcysteine (NAC) was obtained from Beyotime (Shanghai, China). GMEE (sc-203974), AKT inhibitor IV (sc-203809) and AG490 (sc-202046) were purchased from Santa Cruz Biotechnology (Santa Cruz, CA, USA). U0126 (cat. \#9903) was purchased from Cell Signaling Technology (CST; Danvers, MA, USA). Antibodies against phospho-AKT (Ser473), AKT, phospho-ERK1/2 (Thr202/Tyr204), ERK1/2, phospho-STAT3 (Tyr705), STAT3, histone $\mathrm{H} 3$ and $\beta$-actin were purchased from CST. Antibodies against GR, GCLC and Nrf2 were obtained from Santa Cruz Biotechnology.

Cell culture. Human GBM cell lines A172, U87 and U251 were obtained from the Cell Bank of Shanghai, Institute of Biochemistry and Cell Biology, Chinese Academy of Sciences (Shanghai, China). The cells were cultured at $37^{\circ} \mathrm{C}$ in $5 \% \mathrm{CO}_{2} / 95 \%$ air in Dulbecco's modified Eagle's medium (DMEM) containing 10\% heat-inactivated fetal bovine serum (FBS), $100 \mathrm{U} / \mathrm{ml}$ penicillin and $100 \mathrm{mg} / \mathrm{ml}$ streptomycin (all from Gibco, Los Angeles, CA, USA).

Normal human astrocytes (NHAs) were obtained from ScienCell (San Diego, CA, USA) (cat. 1800). NHAs were cultured in astrocyte medium (cat. 1801; ScienCell) and cultured following the manufacturer's instructions.

Lentivirus-mediated RNA interference. For lentivirus-mediated silencing of Nrf2, the lentiviral particles and Polybrene were purchased from GenePharma (Shanghai, China). The short hairpin RNA (shRNA) sequence that effectively targets human Nrf2 was: 5'-GCAGTTCAATGAAGCTCAACT-3'. A non-silencing shRNA, with the scrambled sequence 5'-TTCTC CGAACGTGTCACGT-3', was used as the negative control. The lentiviral particles containing Nrf2-specific shRNA or scrambled shRNA were named Nrf2i or Sc, respectively. Cell transfection was performed as per the manufacturer's instructions. Briefly, the cells were plated in a 6-well plate to reach $30-50 \%$ confluence on the day of transfection. After the addition of Polybrene at $4.5 \mu \mathrm{g} / \mathrm{ml}$, the lentiviral particles were used to transfect the cells. Transfection was continued for $24 \mathrm{~h}$ followed by a $24-\mathrm{h}$ recovery period in complete medium. For the selection of cells with stable transfection, cells were grown under selective pressure by puromycin $(2.0 \mu \mathrm{g} / \mathrm{ml}$; Sigma-Aldrich, St. Louis, MO, USA) for up to 2 weeks.

Quantitative real-time PCR. Total RNA was extracted using a modified TRIzol one-step extraction method (Tiangen, Beijing, China), and then the quality of total RNA was assessed by spectrophotometer analysis (OD260/280:1.8-2.2). An amount 400 ng RNA of total RNA was reverse-transcribed using FirstStrand cDNA Synthesis SuperMix (TransGen Biotech, Beijing, China). The cDNA obtained was amplified immediately using the following primers: for Nrf2, 5'-TCAGCGACGGA AAGAGTATGA-3' and 5'-CCACTGGTTTCTGACTGGA TGT-3'; for NQO1, 5'-ATGGTCGGCAGAAGAGC-3' and 5'-GGAAATGATGGGATTGAAGT-3'; for HO-1, 5'-TCTCC GATGGGTCCTTACACTC-3' and 5'-GGCATAAAGCCCT ACAGCAACT-3'; for GCLC, 5'-AGACATTGATTGTCG CTG-3' and 5'-TGGTCAGACTCATTAGCA-3'; for GR, 5'-TTTGTCTCGGTCTTTCGGGG-3' and 5'-CCAGGATCT ATGGCACCGTC-3'; and for GAPDH, 5'-GAAATCCCATC ACCATCTTC-3' and 5'-GGACTCCACGACGTACTCA-3'. The amplification and data acquisition were carried out on a Real-Time PCR System (Agilent Technologies, Santa Clara, CA, USA) using FastStart Universal SYBR-Green Master (Roche, Mannheim, Germany). The conditions consisted of pre-denaturation at $95^{\circ} \mathrm{C}$ for $10 \mathrm{~min}$, followed by 40 cycles at $95^{\circ} \mathrm{C}$ for $15 \mathrm{sec}$ and $60^{\circ} \mathrm{C}$ for $1 \mathrm{~min}$. The PCR amplification efficiency of each gene was established by means of calibration curves. All samples were analyzed in triplicate. The results were analyzed using the $2^{-\Delta \Delta \mathrm{Cq}}$ method. The data are expressed as the relative mRNA levels and were normalized to GAPDH, a commonly used control.

Western blot analysis. To prepare the total proteins, cells were lysed in cold RIPA lysis buffer containing a $0.5 \%$ phosphatase inhibitor and $1 \%$ phenylmethylsulphonyl fluoride (both from Beyotime, Jiangsu, China). To prepare the nuclear and cytoplasmic proteins, cells were lysed using a nuclear and cytoplasmic protein extraction kit (Beyotime) as per the manufacturer's instructions. Protein content was quantified with the Braford reagent (Coomassie Plus Protein Assay Reagent; cat. \#23238) (Pierce, Rockford, IL, USA). Equal amounts of proteins were separated by $8-15 \%$ sodium dodecyl sulfate polyacrylamide gel electrophoresis and subsequently transferred onto nitrocellulose membranes (Millipore, Billerica, MA, USA). Following blocking with $5 \%$ non-fat milk in TBS-1\% Tween-20 (TBST) for $2 \mathrm{~h}$, the membranes were immunoblotted with primary antibodies: 1:500 anti-Nrf2 (cat. \#sc-13032; 100 kDa), 1:500 anti-GCLC 
(cat. \#sc-22755; $73 \mathrm{kDa}$ ), 1:500 anti-GR (cat. \#sc-32886; 50-65 kDa) (all from Santa Cruz Biotechnology) 1:1,000 anti-phospho-AKT (cat. \#4060; 60 kDa), 1:1,000 anti-AKT (cat. \#4691; $60 \mathrm{kDa}$ ), 1:1,000 anti-phospho-ERK1/2 (cat. \#4370; $42.44 \mathrm{kDa}), 1: 1,000$ anti-ERK1/2 (cat. \#4695; $42.44 \mathrm{kDa}$ ), 1:1,000 anti-phospho-STAT3 (cat. \#9145; $79.86 \mathrm{kDa}$ ), 1:1,000 anti-STA3 (cat. \#12640; $79.86 \mathrm{kDa}$ ), $1: 2,000$ anti- $\beta$-actin (cat. \#4970; $45 \mathrm{kDa}$ ) and 1:1,000 antihistone H3 (cat. \#4499; 17 kDa) (all from CST). After removal of the primary antibodies, the membranes were incubated with the horseradish peroxidase-conjugated secondary antibodies for $2 \mathrm{~h}$ at room temperature and visualized using the enhanced chemiluminescent (ECL) detection reagent from Pierce. Immunoreactive protein bands were detected with Tanon 5200 chemiluminescence imaging system (Shanghai, China).

Doubling time. Aliquots of $5 \times 10^{4}$ cells were seeded in $35-\mathrm{mm}$ plastic dishes in culture medium. The number of cells was counted in triplicate at 24-h intervals for 4 days using a particle distribution counter CDA-500 (Sysmex, Kobe, Japan). The doubling time of the cell population was estimated from the exponential growth phase.

Cell proliferation analysis. Cell proliferation was measured by Cell Counting Kit-8 (CCK-8) (Dojindo, Kumamoto, Japan) as per the manufacturer's protocol. One day prior to treatment, 1,000 cells/well were plated in a 96-well tissue culture plate in a total volume of $200 \mu \mathrm{l} /$ well and were cultured for 1-4 days, respectively. Subsequently, $10 \mu \mathrm{l}$ of CCK-8 solution was added and the cells were further incubated for $1 \mathrm{~h}$ at $37^{\circ} \mathrm{C}$ in $5 \%$ $\mathrm{CO}_{2} / 95 \%$ air. Then, the absorbance was measured at $450 \mathrm{~nm}$ using an ELISA microplate reader (Bio-Rad, Hercules, CA, USA). At least 6 technical replicates were used for each condition.

Colony formation analysis. Colony formation analysis was carried out 9 days after lentivirus transfection. U251 cells were plated into 6-well plates (300 cells/well) and cultured for 9 days. The medium was updated every 3 days. After 9 days of culture, the cells were washed with phosphate-buffered saline (PBS), fixed in 4\% paraformaldehyde for $10 \mathrm{~min}$, and were stained with freshly prepared crystal violet staining solution for $20 \mathrm{~min}$. Colony formation was observed through a light microscope and a colony count was performed.

Assessment of total GSH content. Cells were collected and lysed with protein detergent $\mathrm{S}$ solvent. The total GSH was determined by commercially available Total Glutathione Assay kit (Beyotime Institute of Biotechnology, Shanghai, China). All procedures completely complied to the manufacturer's instructions. The protein concentration was estimated by Coomassie Plus Protein Assay Reagent (Pierce).

Assessment of intracellular ROS. The level of intracellular ROS was quantified using the Reactive Oxygen Species Assay kit (Beyotime Institute of Biotechnology). 2',7'-Dichlorodihydrofluorescein diacetate (DCFH-DA) is oxidized by ROS in viable cells to dichlorofluorescein (DCF) which is highly fluorescent at $530 \mathrm{~nm}$. The cells were washed 3 times with PBS. DCFH-DA, diluted to a final concentration of $10 \mathrm{mM}$, was added and incubated for $30 \mathrm{~min}$ at $37^{\circ} \mathrm{C}$ in the dark. After being washed 3 times with PBS, the relative levels of fluorescence were quantified by a multidetection microplate reader (485 $\mathrm{nm}$ excitation and $535 \mathrm{~nm}$ emission).

Statistical analysis. Data bars correspond to the mean values \pm standard deviations $(\mathrm{SD})$ of minimum triplicate experiments. The statistical significance of the differences was assessed using one-way analysis of variance (ANOVA) or unpaired two-tailed t-tests; the probability values of $<0.05$ and $<0.01$ were deemed statistically significant and very significant, respectively, for all analyses.

\section{Results}

Nrf2 is overexpressed in GBM cell lines. In a previous study, we found that the expression level of Nrf2 was positively correlated with the tumor grade in glioma tissues (23). In the present study, we used western blot analyses to examine Nrf2 levels in various GBM cell lines and normal human astrocyte (NHA) cell line. As shown in Fig. 1A, western blot analysis revealed that all 3 cell lines, U251, U87 and A172, had a higher Nrf2 level than that of the NHA cell line both in the nuclear fraction and in the cytosol fraction. To be noted, there was no detectable band in the nuclear fraction of the NHA cell line, while Nrf2 was accumulated most abundantly in the nuclear fraction of the U251 cells. Consistent with the aforementioned results, similar patterns were observed in the mRNA levels of Nrf2. All three human GBM cell lines (U251, U87 and A172) had increased relative mRNA levels of Nrf2 compared with the NHA cell line (Fig. 1B).

As a transcription factor, overexpression of Nrf2 may cause expression of downstream genes; thus, we tested the mRNA levels of NQO1 and HO-1 using real-time PCR. As shown in Fig. 1B, the highest Nrf2 level was observed in the U251 cells, as well as the mRNA levels of HO-1 and NQO1. Therefore, Nrf2 is overexpressed and transcriptionally active, which may cause overexpression of downstream genes in multiple GBM cell lines.

We also tested the doubling times for U251, U87 and A172 cells $(\sim 8,24$ and $48 \mathrm{~h}$, respectively). It was determined that U251, with the highest Nrf2 expression level, also had the fastest proliferation speed of all 3 cell lines (data not shown). In accordance with this, we chose the U251 cell line for subsequent genetic intervention.

Nrf2 deficiency leads to oxidative stress and redox imbalance in GBM cells. To generate the stable Nrf2-knockdown model, the U251 cells were transfected with lentivirusmediated shRNA ecoding Nrf2-targeting shRNA (Nrf2i) or scrambled control (Sc) shRNA. Clearly, transfection with Sc shRNA did not affect the expression of Nrf2 as the Nrf2 mRNA transcripts and protein expression in the Sc group were similar to that in the control group (Fig. 2A and B). In contrast, the levels of Nrf2 mRNA transcripts in the U251Nrf2i cells were significantly decreased by $\sim 80 \%(\mathrm{P}<0.01)$ (as shown in Fig. 2A), compared with the non-treated or Sc shRNA-treated U251 cells. Additionally, western blot analysis revealed that the $\mathrm{Nrf} 2$ expression was also significantly decreased in the Nrf2i group (Fig. 2B). These results indicated 


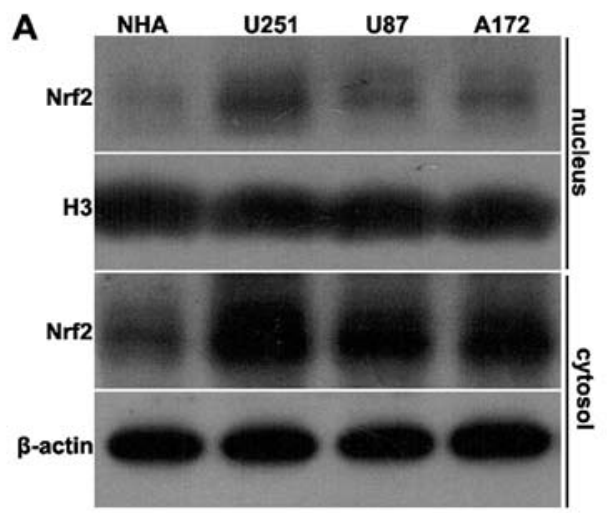

B

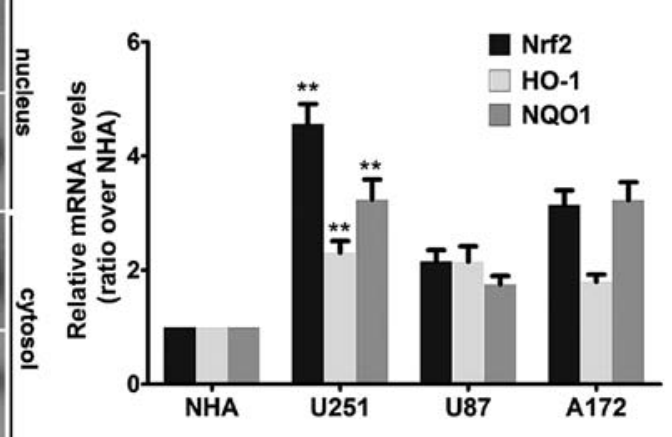

Figure 1. Human GBM cell lines accumulate Nrf2. (A) Western blot analysis of nuclear and cytosolic fractions for Nrf2 protein levels revealed that 3 GBM cell lines (U251, U87 and A172) accumulated activated Nrf2 either in the cytosol or in the nuclei compared with a normal human astrocyte (NHA) cell line. $\beta$-actin and H3 were used as loading controls. (B) Real-time quantitative PCR analysis revealed that human GBM cell lines (U251, U87 and A172) exhibited increased relative mRNA levels of Nrf2 compared with the NHA group. The relative mRNA levels of Nrf2 target genes HO-1 and NQO1 were also increased in comparison with the mRNA levels of Nrf2. Bar graphs were obtained from real-time quantitative PCR analysis and are expressed as ratios over the NHA group, respectively; ** $\mathrm{P}<0.01$ vs. the NHA group.

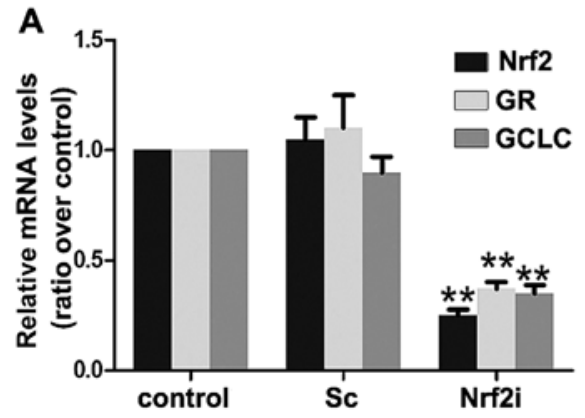

B
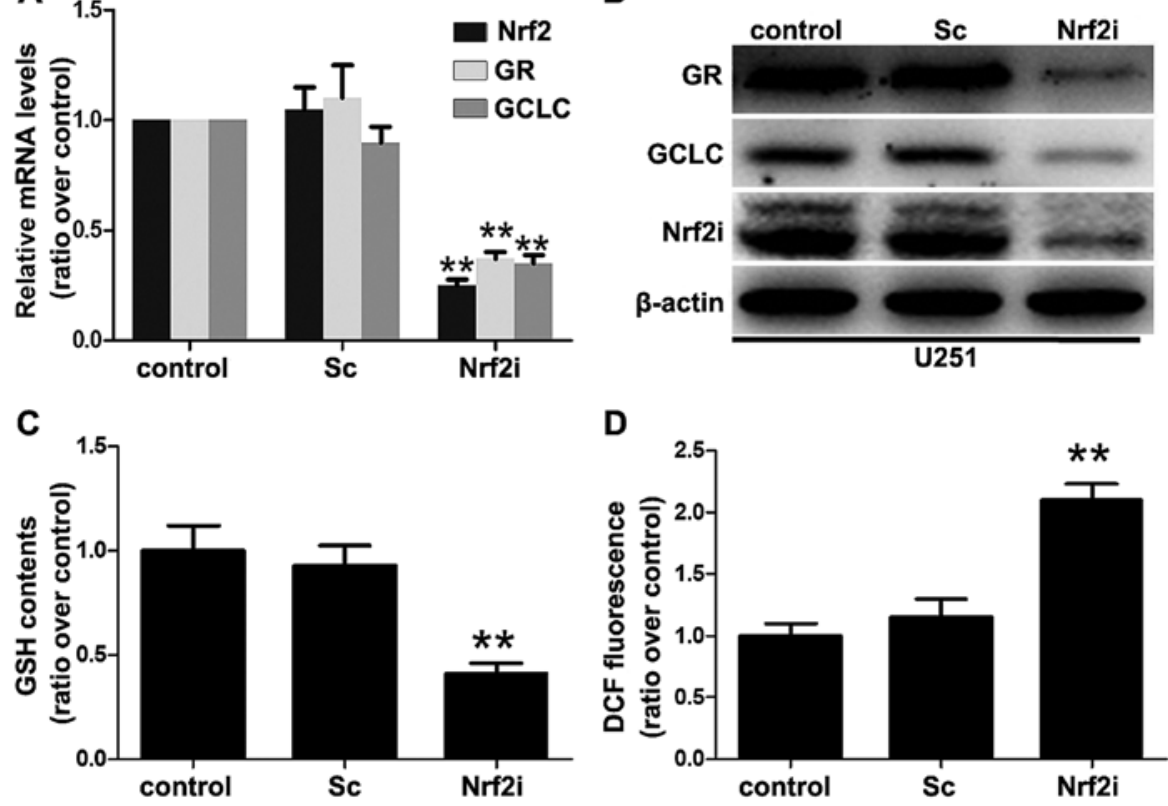

Figure 2. U251 cells deficient in Nrf2 undergo oxidative stress as a result of decreased GSH levels. (A) Relative mRNA levels of Nrf2, GCLC and GR ascertained the inhibition of Nrf2 in U251 cells. Bar graphs were obtained from real-time quantitative PCR analysis and are expressed as the ratios over the control group, respectively; ${ }^{* *} \mathrm{P}<0.01$ vs. the control group. (B) The protein expression of Nrf2, GCLC and GR was determined by western blot analysis using $\beta$-actin as the loading control. (C) GSH levels in non-treated (control), scrambled control (Sc) shRNA and Nrf2 shRNA (Nrf2i)-treated U251 cells were determined. The control value was $20.83 \pm 0.93 \mathrm{nmol} / \mathrm{mg}$; ${ }^{* *} \mathrm{P}<0.01 \mathrm{vs}$. the control group. (D) The relative levels of reactive oxygen species (ROS) in non-treated (control), Sc shRNA and Nrf2 shRNA (Nrf2i)-treated U251 cells. ROS generation was determined using the fluorescence probes DCFH-DA. Values are expressed as the mean $\pm \mathrm{SD}$ from 3 experiments; ${ }^{* *} \mathrm{P}<0.01$ vs. the control group.

that lentivirus-mediated shRNA effectively and specifically suppressed Nrf2 expression in the U251 cells.

As a transcription factor, knockdown of Nrf2 may suppress the expression of its regulated genes, for example GCLC and GR, which are also known as GSH generating enzymes [catalytic subunit of GCL (GCLC) for de novo GSH biosynthesis and GR for GSH regeneration]. Thus, we determined the GCLC and GR mRNA levels using real-time PCR to evaluate the function of Nrf2. As shown in Fig. 2A, the Nrf2 mRNA level was suppressed in the U251 cells accompanied by the decreased mRNA levels of GCLC and GR. Similar patterns were observed in the immunoblot analysis of the proteins GCLC and GR (Fig. 2B).

Since Nrf2 is the GSH-generation key regulator (as indicated above), several in vivo studies have shown that an Nrf2 deficiency results in oxidative stress and redox imbalance (decreased levels of GSH and increased levels of ROS) $(2,3)$. To characterize this effect, we determined the GSH levels in the non-treated, Sc shRNA-treated and Nrf2 shRNA (Nrf2i)-treated cells (Fig. 2C). Consistent with the results obtained with decreased GCLC and GR expression, we found that the GSH levels in the Nrf2-knockdown U251 cells were 
A

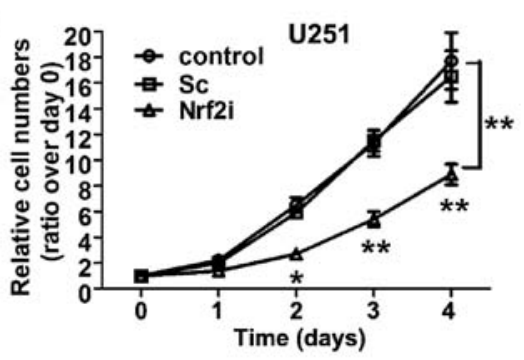

C

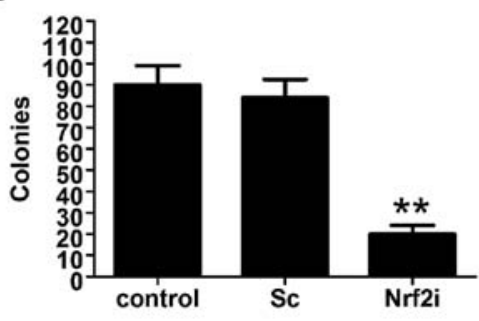

B

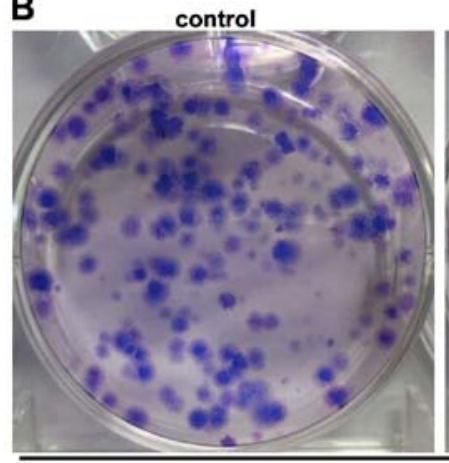

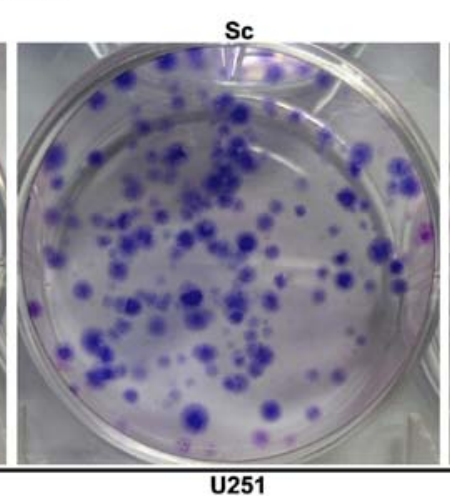

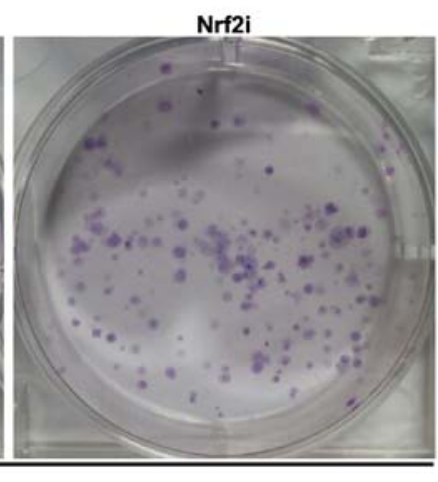

Figure 3. U251 cell proliferation is decreased following the disruption of Nrf2. (A) Cells were cultured for 4 days and cell proliferation was assessed every $24 \mathrm{~h}$ by CCK-8 assay; ${ }^{\mathrm{P}}<0.05,{ }^{* *} \mathrm{P}<0.01$ vs. the control group. (B) The size and number of colonies in the non-treated (control), scrambled control (Sc) shRNA and Nrf2-shRNA (Nrf2i)-treated U251 cells were assessed and recorded under a light microscope. The representative images are from one of 3 independent experiments performed. (C) Statistical analysis of the number of colonies in the control, Sc and Nrf2i groups. Data are presented as the mean \pm SD of 3 independent experiments; ${ }^{*} \mathrm{P}<0.01$ as compared with the control group.

significantly decreased (decreased by $70 \%$ compared with the $\mathrm{Sc}$ and control groups). To further correlate these results with those related to cellular redox status, we determined the intracellular levels of ROS using DCF-DA dye, which emits green fluorescence in the presence of ROS. As shown in Fig. 2D, U251-Nrf2i cells exhibited a $117.4 \%$ increase in ROS production in comparison to the control group (Fig. 2D). Collectively, these data indicated that lentivirus-mediated shRNA silencing led to oxidative stress as a result of decreased GSH levels and redox imbalance in the GBM cells.

U251 cell proliferation is impaired with the disruption of $N r f 2$. To investigate the effect of Nrf2 silencing on cell proliferation, the cell viability was observed using CCK- 8 assay. As shown in Fig. 3A, the growth curve of the Nrf2i group (Nrf2 shRNA-treated U251 cells) began to decline from the second day, compared with the control group (non-treated) and Sc group (Sc-treated U251 cells). The decrease reached $37.1 \%(\mathrm{P}<0.01)$ and $45.2 \%(\mathrm{P}<0.01)$ on the third and fourth day, respectively, compared with the Sc and control groups, whereas no significant difference relating to cell viability was observed between the Sc and control groups. To further ascertain the results of the CCK- 8 assay, we conducted the colony formation assay to evaluate the long-term effect of Nrf2 knockdown on U251 cell proliferation. As shown in Fig. 3B, the size of the independent colonies was much smaller in the Nrf2i group than that in the control group and Sc group. Furthermore, the number of colonies that formed in the Nrf2i group was significantly decreased $(\mathrm{P}<0.01)$, compared with the control and Sc groups (Fig. 3C). The aforementioned data indicate that Nrf2 knockdown evidently impaired the proliferation of U251 cells.
Exogenous supplementation with GMEE, but not NAC, rescues the phenotypic defects associated with Nrf2 deficiency. To further confirm the mechanism of Nrf2 knockdown-induced inhibition of GBM cell proliferation, we wondered whether exogenous supplementation of the Nrf2-knockdown cells with NAC or GMEE (a membrane-permeable derivative of GSH that can supplement cellular GSH levels) may restore their intracellular redox status and cell proliferation. When Nrf2knockdown cell cultures were supplemented on day 1 to day 4 with NAC, GMEE or vehicle (PBS) at various concentrations (see Fig. 4A and B), we found that supplementation with GMEE, but not NAC, restored cell proliferation in the Nrf2knockdown cells for the most part, although the treated cells did not reach the same levels in proliferation as was observed in the control and Sc groups (Fig. 4B). GMEE supplementation also restored the cellular GSH levels that were decreased in the Nrf2-deficient U251 cells (Fig. 4C). Notably, we found that supplementation of Nrf2-deficient cells with NAC, a precursor of GSH, did not improve cell proliferation (Fig. 4A), even though it completely eliminated the high levels of ROS that were otherwise present in the Nrf2-knockdown cells (Fig. 4D). These results collectively suggest that, in addition to squelching ROS, intracellular GSH levels are critical for the proliferation of Nrf2-knockdown cells.

AKT signaling is critical for GMEE-induced Nrf2-deficient U251 cell proliferation. To further delineate the mechanisms by which Nrf2 regulates cell proliferation, we examined the status of intracellular signal transduction pathways, such as AKT, ERK1/2 and STAT3, which are known to be important in cell proliferation. Since exogenous supplementation of Nrf2-deficient cells with GMEE did not increase cell 
A

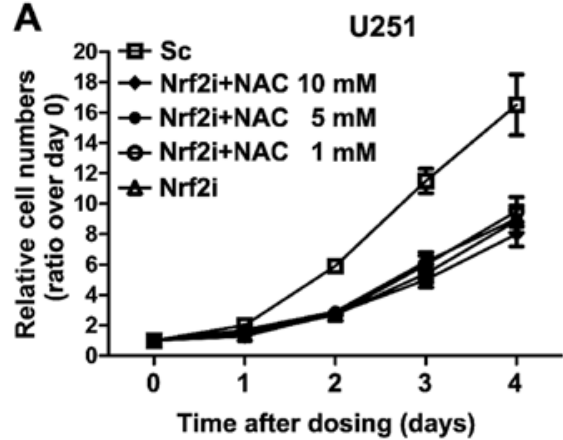

C

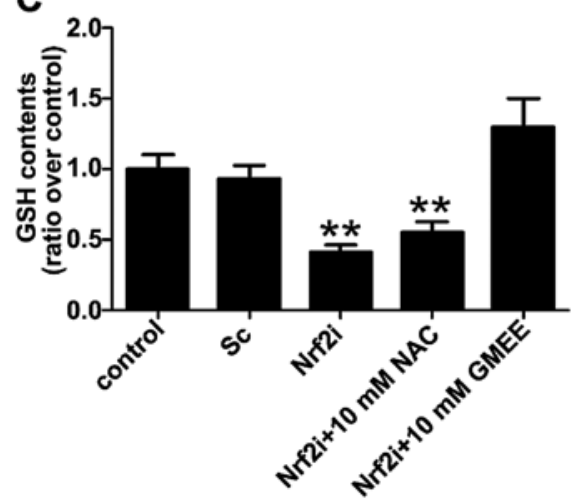

B

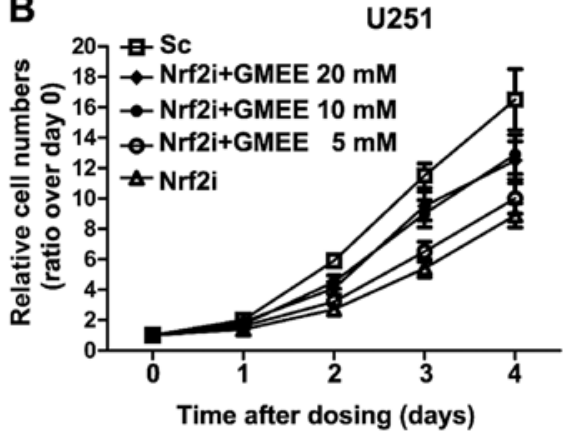

D

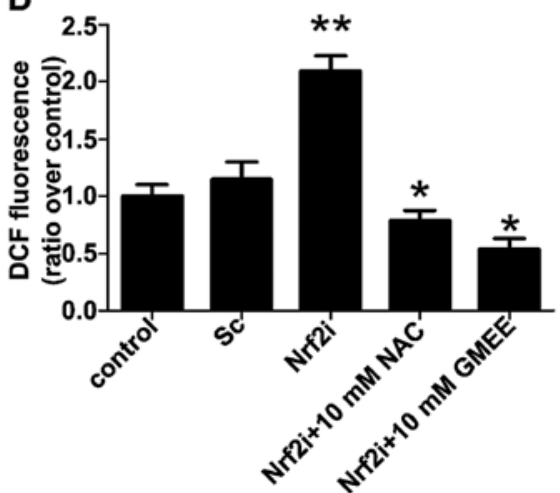

Figure 4. Exogenous supplementation with glutathione monoethyl ester (GMEE) restores Nrf2 deficiency-related phenotypic defects such as cell proliferation. (A and B) Supplementation with GMEE, but not NAC, mostly restored the arrest in cell proliferation noted in the Nrf2-knockdown cells, although the treated cells did not reach the same level as was observed in the Sc group. Cells were grown in the absence or presence of GMEE (5, 10 and 20 mM) and NAC (1, 5 and $10 \mathrm{mM}$ ) for 4 days and cell proliferation was assessed every 24 h by CCK-8 assay. (C) Supplementation of Nrf2-deficient U251 cells with GMEE, but not NAC, restored normal GSH levels that were decreased in the Nrf2-knockdown cells. Cells were grown in the absence or presence of 10 mM GMEE or $10 \mathrm{mM}$ NAC. On day 4 the cells were washed with PBS, lysed and the GSH levels were determined; ${ }^{* *} \mathrm{P}<0.01$ as compared with PBS (vehicle)-treated control group. (D) Following supplementation of Nrf2-deficient U251 cells with GMEE or NAC, both were able to eliminate the increased levels of ROS that were present in the Nrf2-knockdown cells. Cells were grown in the absence or presence of $10 \mathrm{mM}$ GMEE or $10 \mathrm{mM}$ NAC. On day 4 the cells were washed with PBS, lysed and the relative levels of reactive oxygen species (ROS) generation were determined using the fluorescence probes DCFH-DA. Values are expressed as the mean \pm SD from 3 experiments; ${ }^{*} \mathrm{P}<0.05,{ }^{* *} \mathrm{P}<0.01$ as compared with the PBS (vehicle)-treated control group.

proliferation to the same extent as was observed in the counterpart (as shown in Fig. 4B), we reasoned that knockdown of Nrf2 in U251 cells may cause the dysregulation of a series of signal transduction pathways, but GMEE can only restore some, not all of them. In the present study, we analyzed the phosphorylation status and protein levels of AKT, ERK1/2 and STAT3 kinases in the Nrf2-deficient cells with or without GMEE supplementation by immunoblotting. As shown in Fig. 5A, Nrf2 depletion decreased the phosphorylation status as well as the protein levels of AKT. Nrf2 knockdown also significantly decreased the phosphorylation, but not the level of expression of ERK1/2 kinases (Fig. 5A), whereas the phosphorylation status and the protein levels of STAT3 exhibited no evident alteration in the Nrf2-deficient U251 cells. Moreover, the phosphorylation and expression levels of AKT kinase were restored when the Nrf2-deficient U251 cell cultures were supplemented with GMEE. However, the GMEE supplementation failed to restore the phosphorylation status of ERK1/2 kinases that were also dysregulated in the Nrf2-deficient cells. Collectively, Nrf2 deficiency caused the inhibition of the AKT and ERK1/2 pathways in U251 cell lines, but exogenous supplementation of GMEE only restored AKT but not ERK1/2 kinases.

To further verify the results of western blotting, we performed a set of experiments with the AKT-specific inhibitor, AKT inhibitor IV; the ERK inhibitor, U0126; and the STAT3 inhibitor, AG490. AKT inhibitor IV $(5 \mu \mathrm{M})$, U0126 $(10 \mu \mathrm{M})$ and AG490 $(10 \mu \mathrm{M})$ were used to interrupt the signaling transduction of AKT, ERK1/2 and STAT3 at concentrations that did not affect the cell viability when applied alone (data not shown). We found that inhibition of ERK1/2 and STAT3 had no significant effect on GMEE-induced Nrf2-deficient cell proliferation (Fig. 5C and D). In contrast, blocking AKT signaling with an AKT-specific inhibitor markedly blocked the Nrf2-deficient cell proliferation induced by GMEE (Fig. 5B). Collectively, these results strongly suggest that AKT signaling is important in GMEE-induced Nrf2-deficient cell proliferation.

\section{Discussion}

In the present study, we observed the effects of Nrf2 knockdown on cell proliferation, intracellular redox status and the functions of cellular signal transduction pathways. We found that Nrf2 deficiency led to a decrease in U251 cell proliferation and caused intracellular redox imbalance (decreased levels of GSH and increased levels of ROS). Moreover, Nrf2 depletion also resulted in the inhibition of AKT and ERK1/2 signaling. We further found that GMEE supplementation reversed the Nrf2 deficient-induced cell growth arrest and restored intracellular GSH levels. In addition, GMEE supplementation also restored AKT signaling 


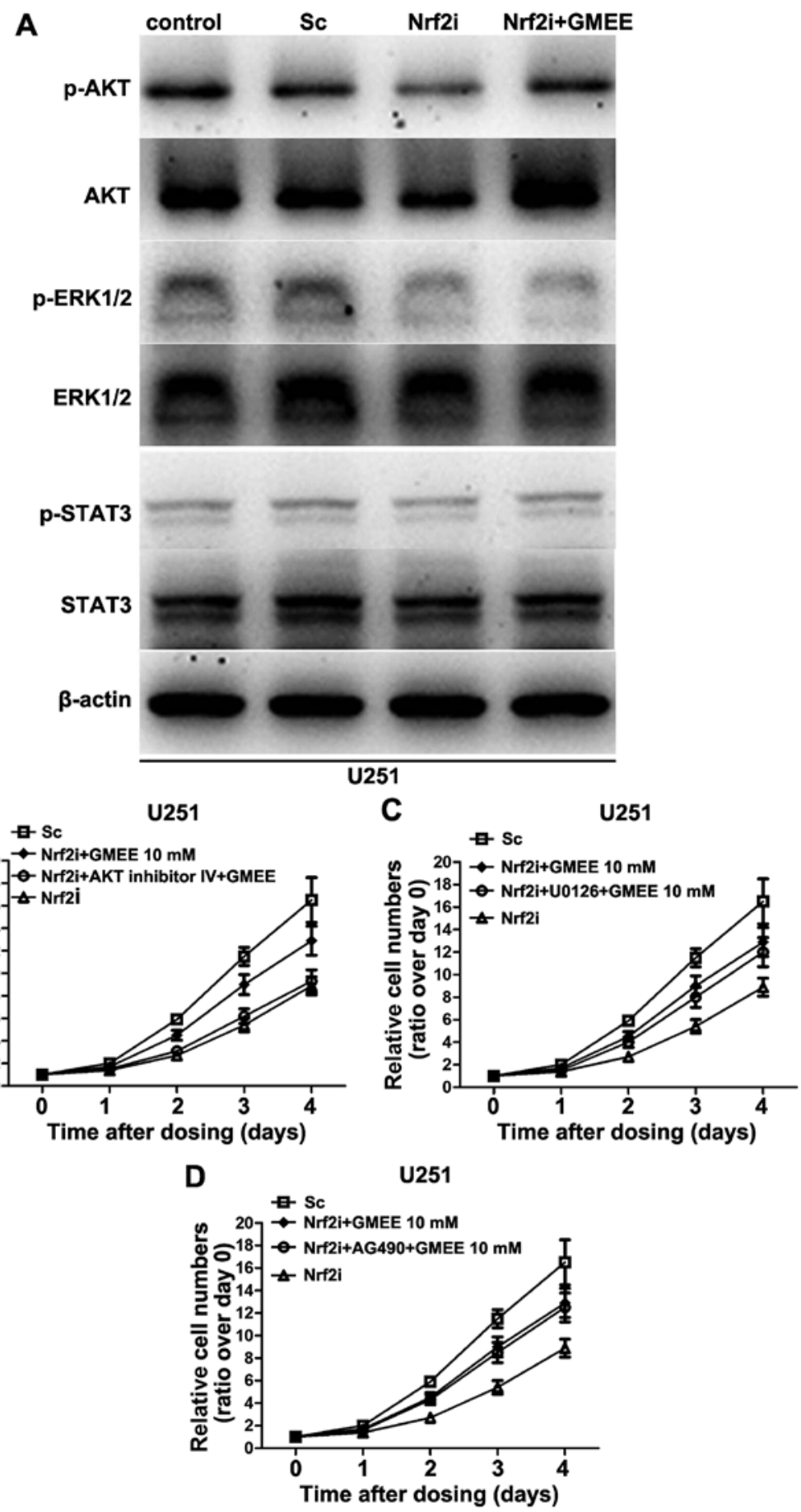

Figure 5. AKT kinase mediates the GMEE-induced Nrf2-deficient U251 cell proliferation. (A) Disruption of Nrf2 resulted in dysfunctional AKT and ERK1/2 signaling, while GMEE supplementation only restored AKT activation. Cells were grown in the absence or presence of $10 \mathrm{mM}$ GMEE or $10 \mathrm{mM}$ NAC for 4 days. On day 4 cell lysates from the non-treated (control), scrambled control (Sc) shRNA and Nrf2-shRNA (Nrf2i)-treated U251 cells were prepared and immunoblotted with antibodies specific for AKT, ERK1/2 and STAT3. $\beta$-actin was used as a loading control. (B-D) Inhibition of ERK1/2 and STAT3 had no significant effects on GMEE-induced Nrf2-deficient cell proliferation (C and D). In contrast, blocking AKT signaling with an AKT-specific inhibitor markedly blocked the Nrf2-deficient cell proliferation induced by GMEE (B). Nrf2-deficient U251 cells were incubated with either GMEE (10 mM) alone or respectively combined with the AKT inhibitor (AKT inhibitor IV, $5 \mu \mathrm{M}$ ), ERK1/2 inhibitor (U0126, $10 \mu \mathrm{M}$ ) and STAT3 inhibitor (AG490, $10 \mu \mathrm{M})$ for 4 days. Cell viability was assessed every $24 \mathrm{~h}$ by CCK- 8 assay.

but not ERK1/2 signaling, although they were both suppressed in the Nrf2-knockdown U251 cells. Notably, we unexpectedly found that supplementation of cells with antioxidant NAC appreciably decreased ROS levels, but it failed to counteract the phenotypic defects associated with cell proliferation. Finally, we provided evidence that the inhibition of ERK1/2 and STAT3 had no significant effect on GMEE-induced
Nrf2-deficient cell proliferation. In contrast, blocking AKT signaling with an AKT-specific inhibitor markedly blocked the Nrf2-deficient cell proliferation induced by GMEE, confirming that AKT signaling is important in GMEEinduced Nrf2-deficient cell proliferation. In the present study, we first revealed that Nrf2 knockdown induced inhibition of proliferation possibly by GSH depletion and consequent AKT 
signaling inhibition, a new perspective by which to understand the role of Nrf2 activation in cancer pathogenesis.

Of particular note is our observation that supplementation of Nrf2-knockdown cells with the antioxidant $\mathrm{N}$-acetylcysteine (NAC), a precursor of GSH, markedly lowered ROS levels but failed to induce cell proliferation. Several studies have shown that NAC attenuates or provides protection against prooxidant stimuli both in vitro and in vivo $(24,25)$. Consistent with these results, our experiments also demonstrated that NAC could markedly squelch ROS levels, but it was unable to restore the decreased intracellular GSH levels that were present in Nrf2-knockdown U251 cells. The inability of NAC to restore the decreased GSH levels in Nrf2-knockdown cells is not surprising, since these cells expressed decreased levels of the $\gamma$-glutamyl cysteinyl ligase (GCL) components GCLC and glutathione reductase (GR), whose activities are essential for GSH generation from its precursor NAC (26-28). Although the sources contributing to the high levels of ROS in Nrf2knockdown cells remain to be investigated, it is likely that decreased levels of GSH may lead to an inefficient cellular detoxification of endogenous ROS levels generated by mitochondria constitutively in Nrf2-silenced cells. In addition, it is possible that Nrf2 deficiency may result in dysregulation of ROS generating enzymes, such as Nox/Duox, Rac or RAS, thereby contributing to increased levels of ROS. Based on the aforementioned results, we directly treated the Nrf2-deficient cells with GMEE (a membrane-permeable derivative of GSH that can supplement cellular GSH levels) in culture medium. As expected, in addition to squelching the high levels of ROS, GMEE supplementation reversed Nrf2 deficiency-induced cell growth arrest and restored the intracellular GSH levels. Thus, it is likely that Nrf2-knockdown cells may only inefficiently convert NAC to GSH, a process that may otherwise cause the intracellular GSH to rise to levels that are adequate for cell proliferation.

To further define the mechanisms contributing to the growth arrest caused by Nrf2 deficiency, we assessed the status of the cellular signaling transduction pathways, since we reasoned that decreased GSH levels in Nrf2-knockdown cells may cause a dysregulation of signal transduction pathways that are related to cell proliferation. This notion is based on recent studies that have suggested a role for protein glutathionylation, a reversible post-translational mechanism involved in the modulation of the activities of the redoxsensitive thiol proteins, particularly those involved in signal transduction and cell proliferation (29). In the present study, we found that suppression of Nrf2 expression could lead to AKT and ERK1/2 pathway inhibition (the phosphorylation status of STAT3 exhibited no significant alterations), which are both known to be important in cell proliferation. However, treatment of Nrf2-knockdown cells with GMEE revealed that exogenous supplementation with GMEE could only restore AKT signaling but not ERK1/2 signaling, even though they were both suppressed in the Nrf2-knockdown U251 cells. Knockdown of Nrf2 in U251 cells caused the dysfunction of a series of signal transduction pathways, but GMEE supplementation only restored them in part, not completely. These observations could be used to explain the results obtained as indicated in Fig 4 which revealed that exogenous supplementation of Nrf2-deficient cells with GMEE did not induce cell proliferation to the same extent as was observed in the counterparts.

To further confirm these results, we finally provided evidence that the inhibition of ERK1/2 and STAT3 had no significant effects on GMEE-induced Nrf2-deficient cell proliferation, whereas, blocking AKT signaling with an AKT-specific inhibitor markedly blocked the Nrf2-deficient cell proliferation induced by GMEE, confirming that AKT signaling is important in GMEE-induced Nrf2-deficient cell proliferation. Our data are consistent with a previous study (30) in which Reddy et al found that Nrf2 deficiency impaired AKT signaling in primary alveolar epithelial cultures isolated from $\mathrm{Nrf}^{-/-}$mice. However, this research group did not point out the precise crosstalk mechanism between AKT signaling and Nrf2. In the present study, we provide evidence of the crosstalk between these two pathways suggesting that protein (AKT kinase) de-glutathionylation (induced by intracellular decreased GSH levels) may be involved in the AKT inhibition resulting from Nrf2 disruption.

Emerging studies have pointed out that Nrf2 and its downstream target genes play a potential role in tumorigenesis, but the detailed underlying mechanism of the biological effects of Nrf2 activation in cancer remain elusive. In the present study, we revealed the decrease in Nrf2-impaired GBM cell proliferation via GSH depletion and consequent AKT signaling inhibition. For the first time the underlying mechanism of Nrf2 in cancer cell proliferation from the perspective of cellular metabolism, which enhances our comprehension of the role of Nrf2 in tumorigenesis was elucidated. More significantly, knowledge of the association of Nrf2 and cellular metabolismrelated signaling pathways in cancer cells, may provide new strategies for glioma clinical intervention, particularly in the absence of effective Nrf2 inhibitors.

\section{Acknowledgements}

The present study was supported by grants from the National Nature Science Foundation of China (nos. 81371357 and 81402072), and the China Postdoctoral Science Foundation Funded Project (nos. 2014M562665 and 2015M572716).

\section{References}

1. Jolly C and Morimoto RI: Role of the heat shock response and molecular chaperones in oncogenesis and cell death. J Natl Cancer Inst 92: 1564-1572, 2000.

2. Singh KK: Mitochondrial dysfunction is a common phenotype in aging and cancer. Ann NY Acad Sci 1019: 260-264, 2004.

3. Mena S, Ortega A and Estrela JM: Oxidative stress in environmental-induced carcinogenesis. Mutat Res 674: 36-44, 2009.

4. Gottlieb E and Tomlinson IP: Mitochondrial tumour suppressors: A genetic and biochemical update. Nat Rev Cancer 5: 857-866, 2005.

5. Motohashi $\mathrm{H}$ and Yamamoto M: Nrf2-Keap1 defines a physiologically important stress response mechanism. Trends Mol Med 10: 549-557, 2004.

6. Gañán-Gómez I, Wei Y, Yang H, Boyano-Adánez MC and García-Manero G: Oncogenic functions of the transcription factor Nrf2. Free Radic Biol Med 65: 750-764, 2013.

7. Malhotra D, Portales-Casamar E, Singh A, Srivastava S, Arenillas D, Happel C, Shyr C, Wakabayashi N, Kensler TW, Wasserman WW, et al: Global mapping of binding sites for Nrf2 identifies novel targets in cell survival response through ChIP-Seq profiling and network analysis. Nucleic Acids Res 38: 5718-5734, 2010 
8. Wild AC, Moinova HR and Mulcahy RT: Regulation of gammaglutamylcysteine synthetase subunit gene expression by the transcription factor Nrf2. J Biol Chem 274: 33627-33636, 1999.

9. Sasaki H, Sato H, Kuriyama-Matsumura K, Sato K, Maebara K, Wang $H$, Tamba $M$, Itoh $K$, Yamamoto $M$ and Bannai $S$ : Electrophile response element-mediated induction of the cystine/glutamate exchange transporter gene expression. J Biol Chem 277: 44765-44771, 2002.

10. Itoh K, Chiba T, Takahashi S, Ishii T, Igarashi K, Katoh Y, Oyake T, Hayashi N, Satoh K, Hatayama I, et al: An Nrf2/small Maf heterodimer mediates the induction of phase II detoxifying enzyme genes through antioxidant response elements. Biochem Biophys Res Commun 236: 313-322, 1997.

11. Ishii T, Itoh K, Takahashi S, Sato H, Yanagawa T, Katoh Y, Bannai S and Yamamoto M: Transcription factor Nrf2 coordinately regulates a group of oxidative stress-inducible genes in macrophages. J Biol Chem 275: 16023-16029, 2000.

12. Aoki Y, Hashimoto AH, Amanuma K, Matsumoto M, Hiyoshi K, Takano H, Masumura K, Itoh K, Nohmi T and Yamamoto M: Enhanced spontaneous and benzo $(a)$ pyrene-induced mutations in the lung of Nrf2-deficient gpt delta mice. Cancer Res 67: 5643-5648, 2007.

13. Ramos-Gomez M, Kwak MK, Dolan PM, Itoh K, Yamamoto M, Talalay P and Kensler TW: Sensitivity to carcinogenesis is increased and chemoprotective efficacy of enzyme inducers is lost in $n r f 2$ transcription factor-deficient mice. Proc Natl Acad Sci USA 98: 3410-3415, 2001.

14. Lau A, Villeneuve NF, Sun Z, Wong PK and Zhang DD: Dual roles of Nrf2 in cancer. Pharmacol Res 58: 262-270, 2008.

15. Hayes JD and McMahon M: NRF2 and KEAP1 mutations: Permanent activation of an adaptive response in cancer. Trends Biochem Sci 34: 176-188, 2009.

16. Hayes JD and McMahon M: The double-edged sword of Nrf2: Subversion of redox homeostasis during the evolution of cancer. Mol Cell 21: 732-734, 2006.

17. Kensler TW and Wakabayashi N: Nrf2: Friend or foe for chemoprevention? Carcinogenesis 31: 90-99, 2010.

18. Homma S, Ishii Y, Morishima Y, Yamadori T, Matsuno Y, Haraguchi N, Kikuchi N, Satoh H, Sakamoto T, Hizawa N, et al: Nrf2 enhances cell proliferation and resistance to anticancer drugs in human lung cancer. Clin Cancer Res 15: 3423-3432, 2009.

19. Yamadori T, Ishii Y, Homma S, Morishima Y, Kurishima K, Itoh K, Yamamoto M, Minami Y, Noguchi M and Hizawa N Molecular mechanisms for the regulation of Nrf2-mediated cell proliferation in non-small-cell lung cancers. Oncogene 31: 4768-4777, 2012
20. Lister A, Nedjadi T, Kitteringham NR, Campbell F, Costello E, Lloyd B, Copple IM, Williams S, Owen A, Neoptolemos JP, et al: Nrf2 is overexpressed in pancreatic cancer: Implications for cell proliferation and therapy. Mol Cancer 10: 37, 2011.

21. Ji XJ, Chen SH, Zhu L, Pan H, Zhou Y, Li W, You WC, Gao CC, Zhu JH, Jiang K, et al: Knockdown of NF-E2-related factor 2 inhibits the proliferation and growth of U251MG human glioma cells in a mouse xenograft model. Oncol Rep 30: 157-164, 2013.

22. Pan H, Wang H, Zhu L, Mao L, Qiao L and Su X: The role of Nrf2 in migration and invasion of human glioma cell U251. World Neurosurg 80: 363-370, 2013

23. Ji X, Wang H, Zhu J, Zhu L, Pan H, Li W, Zhou Y, Cong Z, Yan F and Chen S: Knockdown of Nrf2 suppresses glioblastoma angiogenesis by inhibiting hypoxia-induced activation of HIF-1 $\alpha$. Int J Cancer 135: 574-584, 2014.

24. Thimmulappa RK, Lee H, Rangasamy T, Reddy SP, Yamamoto M, Kensler TW and Biswal S: Nrf2 is a critical regulator of the innate immune response and survival during experimental sepsis. J Clin Invest 116: 984-995, 2006.

25. Rangasamy T, Guo J, Mitzner WA, Roman J, Singh A, Fryer AD, Yamamoto M, Kensler TW, Tuder RM, Georas SN, et al: Disruption of $N r f 2$ enhances susceptibility to severe airway inflammation and asthma in mice. J Exp Med 202: 47-59, 2005.

26. Hutter DE, Till BG and Greene JJ: Redox state changes in density-dependent regulation of proliferation. Exp Cell Res 232: 435-438, 1997.

27. Pani G, Colavitti R, Bedogni B, Anzevino R, Borrello S and Galeotti T: A redox signaling mechanism for density-dependent inhibition of cell growth. J Biol Chem 275: 38891-38899, 2000.

28. Menon SG, Sarsour EH, Spitz DR, Higashikubo R, Sturm M, Zhang $\mathrm{H}$ and Goswami PC: Redox regulation of the $\mathrm{G}_{1}$ to $S$ phase transition in the mouse embryo fibroblast cell cycle. Cancer Res 63: 2109-2117, 2003.

29. Dalle-Donne I, Carini M, Vistoli G, Gamberoni L, Giustarini D, Colombo R, Maffei Facino R, Rossi R, Milzani A and Aldini G: Actin Cys374 as a nucleophilic target of $\alpha, \beta$-unsaturated aldehydes. Free Radic Biol Med 42: 583-598, 2007.

30. Reddy NM, Kleeberger SR, Bream JH, Fallon PG, Kensler TW, Yamamoto $\mathrm{M}$ and Reddy SP: Genetic disruption of the Nrf2 compromises cell-cycle progression by impairing GSH-induced redox signaling. Oncogene 27: 5821-5832, 2008. 